\title{
Networked Monitoring of Urban Traffic Emissions
}

\author{
Zerui Li, Yu Kang, Ji Chang, and Wenjun Lv
}

\begin{abstract}
This paper presents some key points for networked monitoring of urban traffic emissions. Firstly, we introduce the remote sensing devices distributed on the urban roads to measure the emissions of passing vehicles. Secondly, we discuss how to determine the device locations by maximizing the amount of the captured vehicles. Finally, a method to identify high-emitting vehicles is proposed based on broad learning system. The experiment results show the networked monitoring system is able to inspect massive on-road vehicles in a short-term and accurately identify high-emitting vehicles.
\end{abstract}

Index Terms-High-emitter identification, location strategy, networked monitoring, vehicle emission.

\section{INTRODUCTION}

With the development of modern city, the sharp increase in vehicle population has rendered a lot of traffic problems. Traffic emission rendered by the tremendous amount of vehicles are becoming a primary contributor of urban air pollution, resulting in various adverse effects on human health. The traffic emission induced social issues is rising in many cities of China, and thereby attracting public attention increasingly [1].

In order to alleviate the issues caused by traffic emission, it is crucial to control vehicle emissions in urban environment. The prerequisite of control is monitoring of emissions. Since the large quantity of vehicles and resulting massive emission data, the monitoring needs to be of large scale, low cost, automation, being friendly to drivers and managers. A promising technique to meet all the requirements is remote sensing that quantifies the pollutants from vehicles based on the spectroscopy theory. The remote sensing devices can work 24 hours every day without obstructing the traffic, so thousands of vehicles can be measured by a device in a single day. As a result, remote sensing can make a general survey of all on-road vehicles and screen out high-emitters among them. High-emitters are responsible for almost 80 percent of the pollution caused by all vehicles, although the amount of them is about 20 percent of the vehicle population. Therefore, remote sensing technique is quite a helpful tool for vehicle

Manuscript received October 9, 2018; revised February 3, 2019. This work was supported in part by the National Natural Science Foundation of China under Grant 61725304, Grant 61673361, and Grant 61673350, in part by the Youth Top-Notch Talent Support Program, and in part by the Youth Yangtze River Scholar. (Corresponding author: Yu Kang.)

Z. Li, J. Chang, and W. Lv are with the Department of Automation, University of Science and Technology of China, Hefei 230027, China (e-mail: 1zerui@mail.ustc.edu.cn, wlv@ustc.edu.cn, cjchange@mail.ustc.edu.cn).

Y. Kang is with the State Key Laboratory of Fire Science, Department of Automation and Institute of Advanced Technology, University of Science and Technology of China, Hefei 230027, China. He is also with the Key Laboratory of Technology in GeoSpatial Information Processing and Application System, Chinese Academy of Sciences, Beijing 100190, China (e-mail: kangduyu@ustc.edu.cn). emission control.

Using remote sensing technology to examine on-road vehicle emissions has been studied and applied in the last several decades. In the 1980s, Bishop et al. from University of Denver first developed a long-path IR photometer to measure $\mathrm{CO}$ when vehicles are up and running [2]. In the following years, scientists from University of Denver made a further investigation on the remote sensing technology. By 1996, their system already realized the measurement for CO, $\mathrm{CO} 2, \mathrm{HC}$, NO and smoke opacity [3], [4]. In 2004, experiments in Oregon prove the stability and accuracy of remote sensing technology by comparing and evaluating the measurements of remote sensing and other detection means. Due to the property of low cost, automation and real-time, remote sensing has been successfully applied in many countries mainly as an effective tool to screen out high-emitters, and to identify the effectiveness of the inspection and maintenance program.

Although remote sensing technique has been applied to the real-world traffic network to measure emissions from passing vehicles, the application is confined to single-point detection. The emission data obtained by remote sensing devices cannot be integrated and then analyzed. In addition, the location of devices are chosen randomly or empirically. Therefore, the existing remote sensing system need to be reformed. In this work, we propose solutions for several issues of vehicle emission monitoring. In our early work [5], the problem of networked monitoring of vehicle emission has been put forward. In this paper, we improve the methods not only for device location but also for data analysis. We propose a location strategy that exploits the trajectory of vehicles traveling on the road network. As for data analysis, we propose a high-emitter identification method based on the broad learning system, which is proven to be effective according to the experimental results.

\section{Remote Sensing Device}

The basic module of vehicle emission monitoring is the detection of vehicle emission. In order to obtain the concentration of harmful gases in vehicle emission when vehicles are in their normal operating condition, the remote sensing technology is developed that measures the emission in a non-contact way and thus does not affect the traveling of vehicles [2]-[4].

We apply the remote sensing device for collecting the concentration data of emitted gases from vehicles moving on the road network. The remote sensing device measures the gas concentration based on the attenuation intensity of infrared and ultraviolet light after passing through vehicle emission. The measurement process applies the Beer-Lambert law, that is,

$$
I(\lambda)=I_{0}(\lambda) e^{-\beta c L},
$$


where $I(\lambda)$ represents the light intensity received by the receiver of the gas detector after traveling $L, I_{0}(\lambda)$ represents the light intensity emitted by the light source. $\beta$ represents the atmospheric attenuation coefficient, and $c$ is the gas concentration.

Since the remote sensing device measures the vehicle emission when the vehicles are up and running, the exhaust plume from vehicles varies with wind and turbulence. The remote sensing device can only determine the ratio of $\mathrm{CO}$, $\mathrm{HC}$ and $\mathrm{NO}$ to $\mathrm{CO}_{2}$.

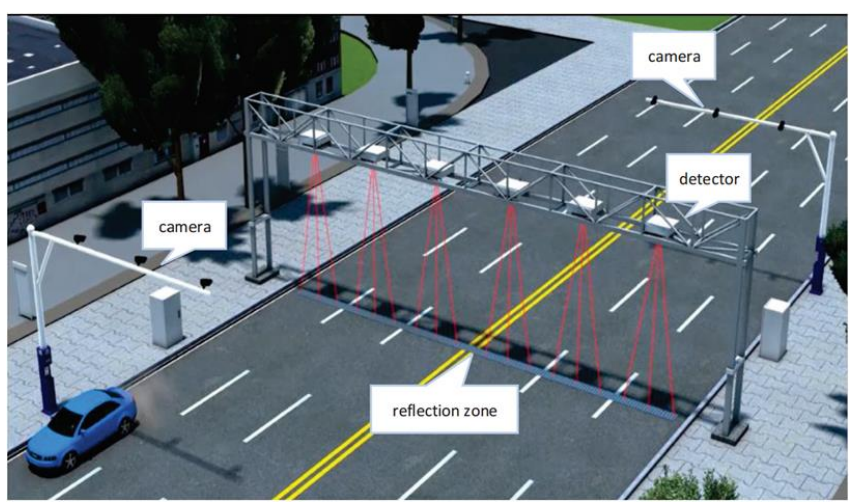

Fig. 1. Structure of the remote sensing device. The detector is the detection module of the device, which mainly includes a gas detector and a speed/acceleration detector. From the figure, it is noticed that the remote sensing device can monitor bidirectional traffic.

Fig. 1 shows that the device mainly consists of a gas detector, a speed/acceleration detector, a reflection zone and several cameras. The cameras are mounted above each lane for monitoring the traffic condition and thus determining the suitable time of detecting. Once the cameras determine the time for detecting, the emitter of the gas detector transmits ultraviolet and infrared lights covering the lane to the reflection zone. Then the receiver of the gas detector receive the lights reflected by the reflection zone. The concentration of $\mathrm{CO}, \mathrm{HC}$ and $\mathrm{NO}$ can be figured out in form of volume ratio according to the light intensity of attenuation. At the same time, the speed/acceleration detector can detect the speed and acceleration of the passing cars according to the time of vehicle passing certain-spaced light beams. Associated with the measurement of the gas detector and the speed/acceleration detector, the measuring conditions like temperature, humidity, wind direction, wind speed are stored in the database for subsequent analysis.

The remote sensing device can work 24/7 and monitor multiple lines simultaneously. As a result the device is of high efficiency and can detect thousands of vehicles every day. Generally speaking, the devices should be allocated to roads with large traffic volume by considerations of cost and efficiency. Since the devices transmit light from above to the ground, and every lane has a corresponding emitter and receiver, the measuring of vehicles on multiple lanes does not interfere with each other. The devices can handle the situation where vehicles pass through the detectors in parallel. As a result, they can be used for roads with dense traffic. However from another aspect, a pair of light emitter and receiver for every lane increases the cost of remote sensing devices, which implies that the number of devices that can be located on the network is much smaller relative to that of road links. Then it is really important where we should locate these devices.

\section{LOCATION OF REMOTE SENSING DEVICES}

As mentioned, locating the limited number of devices on suitable road links is critical for the networked monitoring of vehicle emissions. Then the questions arise: As there are thousands of road links on the network, how can we select the suitable links to locate devices? There is no doubt that the more devices we locate, the better performance we can expect. However any system is constrained by the economic cost. As a result, we consider setting the smallest possible number of devices to capture as many vehicles as possible.

The proposed location strategy in this paper is based on the information of on-road vehicle routes. Consider a traffic network $D=(V, A)$, where $V=\left\{v_{1}, v_{2}, \ldots, v_{m}\right\}$ is the set of vertices representing the intersections on the road network, and $A=\left\{a_{1}, a_{2}, \ldots, a_{n}\right\}$ is the set of road links. Denote a corresponding vector $L=\left\{\ell_{1}, \ell_{2}, \ldots, \ell_{n}\right\} \in\{0,1\}^{n} . \ell_{i}=1$ means that there is a device located on $a_{i} . \ell_{i}=0$ means that there is no device on $a_{i}$. The set of all in-use vehicles is denoted by $C=\left\{c_{1}, c_{2}, \ldots, c_{p}\right\}$ where $p$ is the total amount of in-use vehicles. For vehicle $c_{i}, i=1,2, \ldots, p$, there is a corresponding vector $R_{i} \in\{0,1\}^{n}$. If the trajectory of vehicle $c_{i}$ includes road link $a_{k}, k=1,2, \ldots, n$, the $k$ th element of $R_{i}$ equals to 1 . Otherwise the $k$ th element of $R_{i}$ equals to 0 .

The objective is maximizing the amount of captured vehicles.

$$
\begin{gathered}
\max \sum_{j=1}^{p} \mathbb{I}_{\left\{R_{j}^{\prime} L>0\right\}} \\
\text { subject to } \sum_{i=1}^{n} \ell_{i} \leq M \quad i=1,2, \ldots, n \\
L_{k}=\mathbf{0} \\
\ell_{i} \in\{0,1\} \quad i=1,2, \ldots, n
\end{gathered}
$$

where $L_{k}=\left\{\ell_{k_{1}}, \ell_{k_{2}}, \cdots, \ell_{k_{s}}\right\}$ is the set of elements in $L$ which should be set as 0 because of being unsuitable for locating devices or prohibited to install such devices for the reason of road planning.

$$
\mathbb{I}_{\left\{R_{j}^{\prime} L>0\right\}}= \begin{cases}1, & \text { if } R_{j}^{\prime} L>0 \\ 0, & \text { else }\end{cases}
$$

In order to solve the model above, the hypergraph theory is used. The definitions about hypergraph are explained in detail in [6], so we do not reiterate them here. We establish such a hypergraph $H$ that every vertex of $H$ represents a road link on the road network. We denote the set of vertices by $X$, $X=\left\{x_{1}, x_{2}, \ldots, x_{n}\right\}$. Because the trajectories of vehicles in a certain period are a series of subsets of $X$, we use a hyperedge in $H$ to represent the trajectory of a unique vehicle. Obviously, it is possible that for vehicles $c_{i}$ and $c_{j}$ where $i \neq j$, the corresponding $r_{i}$ and $r_{j}$ are equal to each other. Thus $H$ is not a simple hypergraph. We denote the degree of vertex $x$ by $d(x)$.

Then we solve our problem in this hypergraph. The obvious solution is using the greedy algorithm. That is to say, 
we prefer to choose the vertex in $H$ with larger degree. If we sort the vertices in order of largest degree, in the first step, we will choose the first one that is not in $L_{k}$, say $x_{1}^{*}$. Then all hyperedges that include $x_{1}^{*}$ will be removed from the hypergraph. The degrees of remaining vertices will be updated. The operations above are repeated until $M$ vertices have been chosen or there is no vertex that can be chosen. Finally the chosen vertices are just the road links that should be allocated with remote sensing devices.

\section{DATA ANALYSIS}

It is obvious that we can collect enormous amounts of data from remote sensing devices distributed on the road network. Due to the immunity from interference of adjacent lanes, the ratio of valid data is high. Additionally, the design of device location strategy can further increase the diversity and richness of data and reduce the generation of redundant data. The goal of mounting remote sensing devices is to measure the emission of on-road vehicles and screen high-emitters, and consequently reduce the pollution caused by traffic on the road network. According to the statistical result of the collected data, it is found that only 9.43 percent of valid data is about high-emitters. With the imbalanced dataset, conventional classification methods tend to classify the samples of minority class into the majority class, which causes that the recognition accuracy of minority class is one half to one third of that of majority class [7]. In this paper, we apply the broad learning system to identify high-emitters using data collected by remote sensing devices.

Broad learning system (BLS) is first proposed by Chen and Liu [8]. BLS is an effective and efficient learning system without the deep architecture, which speeds up the training process and also guarantees the classification accuracy. It should be noted that the parameters in the model are of less quantity and can be easily computed in the way of ridge regression approximation. As for more details about BLS, readers can refer to [8].

It is obvious that the identification of high-emitters is based on the concentration of harmful gases $\mathrm{CO}, \mathrm{HC}$ and NO. However the measurement results of remote sensing devices vary with the measuring conditions like temperature, relative humidity, wind speed and direction, and so on. Therefore for establishing the BLS model to identify high emitters, the collected data of remote sensing devices are input to the model, including speed, acceleration, length of vehicle, measured concentration of $\mathrm{CO}_{2}, \mathrm{CO}, \mathrm{HC}$ and $\mathrm{NO}$ in the plume and in the tailpipe, smoke opacity, average opacity, opacity coefficient, and the measurement conditions including wind speed, wind direction, temperature, relative humidity and atmospheric pressure. The output is set to be the measurement results of vehicles whether they emit more emission than the standard or not.

The performance of the BLS model is shown in Table I. In order to eliminate the impact of randomness, we select 5 groups of data randomly, each of which contains 30000 samples. In each group, 25000 samples are randomly chosen as training samples, and the other 5000 samples constitute the test set. In Table I, the precision, recall and F1-measure to evaluate the performance of BLS-based high-emitter identification approach. The values of precision, recall and
F1-measure are all larger than 0.9. It is shown that the approach achieve satisfactory results on all of the 5 datasets.

TABLE I: EXPERIMENTAL RESULTS OF BLS-BASED HIGH-EMITTER IDENTIFICATION

\begin{tabular}{lcccc}
\hline \hline & $\begin{array}{c}\text { Number of } \\
\text { high-emitters }\end{array}$ & Precision & Recall & F1 \\
\hline Group 1 & 1468 & 0.9009 & 1 & 0.9479 \\
\hline Group 2 & 1507 & 0.9643 & 0.9900 & 0.9770 \\
\hline Group 3 & 1389 & 0.9091 & 1 & 0.9524 \\
\hline Group 4 & 1896 & 0.9581 & 0.9900 & 0.9738 \\
\hline Group 5 & 1690 & 0.9673 & 0.9867 & 0.9769 \\
\hline \hline
\end{tabular}

\section{CONCLUSION}

In this paper, we propose solutions for key issues of networked vehicle emission monitoring. First we introduce a remote sensing device that can measure the emissions of passing vehicles in a bidirectional multi-lane road. Then for the location of remote sensing devices on the road network, we design a location strategy based on the trajectory of on-road vehicles. With the hypergraph theory, we transform the location problem of interest into an issue in a hypergraph, that is, finding a subset of the vertex set that intersects as many hyperedges as possible. We solve this problem using the greedy algorithm. At last, we propose a BLS-based high-emitter identification method, and the experiments on real data verify the effectiveness of the method.

\section{REFERENCES}

[1] M. Walsh, "Global trends in motor vehicle pollution control: A 2011 update. part 1," Silniki Spalinowe, vol. 50, pp. 106-117, 2011.

[2] G. A. Bishop, J. R. Starkey, A. Ihlenfeldt, W. J. Williams, and D. H. Stedman, "Ir long-path photometry: A remote sensing tool for automobile emissions," Analytical Chemistry, vol. 61, no. 10, pp. 671A-677A, 1989

[3] Y. Zhang, D. H. Stedman, G. A. Bishop, P. L. Guenther, and S. P. Beaton, "Worldwide on-road vehicle exhaust emissions study by remote sensing," Environmental Science \& Technology, vol. 29, no. 9, pp. 2286-2294, 1995.

[4] G. Bishop and D. Stedman, "Measuring the emissions of passing cars," Accounts of Chemical Research, vol. 29, no. 10, pp. 489-495, 1996.

[5] Y. Kang, Y. Ding, Z. Li, Y. Cao, and Y. Zhao, "A networked remote sensing system for on-road vehicle emission monitoring," Science China Information Sciences, vol. 60, no. 4, p. 043201, 2017.

[6] A. Bretto, "Hypergraph theory," Mathematical Engineering, 2013.

[7] G. M. Weiss and F. Provost, "Learning when training data are costly: The effect of class distribution on tree induction," Journal of Artificial Intelligence Research, vol. 19, pp. 315-354, 2003.

[8] C. P. Chen and Z. Liu, "Broad learning system: An effective and efficient incremental learning system without the need for deep architecture," IEEE Transactions on Neural Networks and Learning Systems, vol. 29, no. 1, pp. 10-24, 2018.

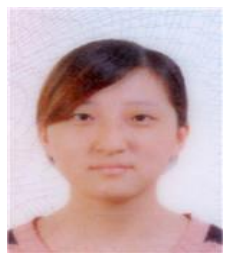

Zerui Li is currently pursuing the Ph.D. degree with the Department of Automation, University of Science and Technology of China, Hefei, China. Her current research interests include vehicle emission remote sensing system, systems engineering and data mining.

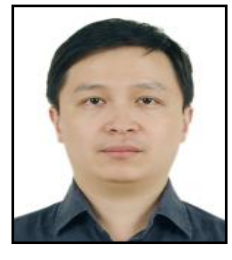

Yu Kang received the Dr.Eng. degree in control theory and control engineering from the University of Science and Technology of China, Hefei, China, in 2005. From 2005 to 2007, he was a post-doctoral fellow with the Academy of Mathematics and Systems Science, Chinese Academy of Sciences, Beijing, China. He is currently a professor with the State Key Laboratory of Fire Science, Department of 
and Institute of Advanced Technology, University of Science and Technology of China and the Key Laboratory of Technology, GeoSpatial Information Processing and Application System, Chinese Academy of Sciences, Beijing, China. His current research interests include monitoring of vehicle emissions, adaptive/robust control, variable structure control, mobile manipulator, and Markovian jump systems.

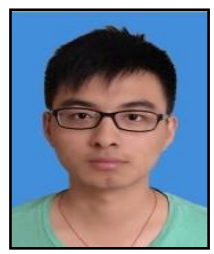

Chang Ji is currently pursuing the master's degree with the Department of Automation, University of Science and Technology of China, Hefei, China. Her current research interests include vehicle emission remote sensing system, and machine learning.

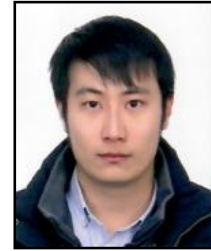

Wenjun Lv received the Ph.D. degree in control science and engineering from the University of Science and Technology of China (USTC), Hefei, China, in 2018. He is currently a postdoctoral fellow with the School of Information Science and Technology, USTC His current research interests include state estimation, machine learning, and their application in robotics. 